\title{
Stereochemical studies on cyclic peptides: Detailed energy minimization studies on hydrogen bonded all-trans cyclic pentapeptide backbones*
}

\author{
H A NAGARAJARAM and C RAMAKRISHNAN $\dagger$ \\ Molecular Biophysics Unit, Indian Institute of Science, Bangalore 560 012, India
}

MS received 20 July 1995; revised 9 October 1995

\begin{abstract}
Conformational studies have been carried out on hydrogenbonded all-trans cyclic pentapeptide backbone- Application of a combination of grid search and energy minimization on this system has resulted in obtaining 23 minimum energy conformations, which are characterized by unique patterns of hydrogen bonding comprising of $\beta-$ and $\gamma$-turns. A study of the minimum energy conformations vis-a-vis non-planar deviation of the peptide units reveals that non-planarity is an inherent feature in many cases. A study on conformational clustering of minimum energy conformations shows that the minimum energy conformations fall into 6 distinct conformational families- Preliminary comparison with available X-ray structures of cyclic pentapeptide indicates that only some of the minimum energy conformations have formed crystal structures. The set of minimum energy conformations worked out in the present study can form a consolidated database of prototypes for hydrogen bonded backbone and be useful for modelling cyclic pentapeptides both synthetic and bioactive in nature-
\end{abstract}

Keywords. Cyclic pentapeptide; conformational studies; reverse turns; $\beta$-turn; $\gamma$-turn; energy minimization.

\section{Introduction}

Cyclic pentapeptides (CPPs) form an interesting class of compounds in the family of cyclic peptides which include naturally occurring compounds as well as the ones obtained by chemical synthesis. Some of them are well known for their activities of biological importance such as antibacterial activity, toxicity, cell growth inhibition, etc., (Ovchinnikov and Ivanov 1982).

It is now a well-recognized fact that CPPs offer convenient systems to model reverse turns (Rose et al 1985; Stradley et al 1990; Rizo et al 1991). The CPP analogues have been found useful in deducing structure-activity relations associated with the bio-active peptides (Kessler 1982) - Accordingly during the past two decades several CPPs with varying sequences have been synthesized and their structures have been elucidated by X-ray crystallography and NMR techniques (Demel and Kessler 1976; Bara et al 1978; Karle 1978, 1979, 1981, 1986; Kessler and Kondor 1979; Kessler and Holzeman 1981; Kessler and Evermann 1982; Mauger et al 1982; Kessler and Koggler 1983; Kessler and Kutscher, 1985; Lautz et al 1987; Kessler et al 1989; Stradley et al 1990; Rizo et al 1991; Liu and Gierasch 1992; Stroup et al 1992; Gonnella et al 1994). These studies have thrown light

\footnotetext{
*This is part XV of the series. Part XIV in this series is Ramakrishnan et al 1987.

${ }^{\dagger}$ Corresponding author (Fax: 091-80-3092715: Email: ramki@mbu·iisc·ernet·in)
} 
on the conformational preferences of the CPP backbone in the solid state and in the solution environments. They reveal that the backbone can adopt one of the following conformational types viz, (i) conformations with only one $\beta$-turn, (ii) conformations having a $\beta$-turn fused to a $\gamma$-turn and (iii) conformations having a pair of $\gamma$-turns.

This group has been involved in the study of intramolecularly hydrogen bonded CPP having all-trans backbone. The three types of hydrogen bonded CPP which have been studied are, (i) $4 \rightarrow 1$ hydrogen bonded $\beta$-type (Manjula and Ramakrishnan 1979), (ii) $4 \rightarrow 1$ and $3 \rightarrow 1$ hydrogen bonded $\beta \gamma$-type (Rao 1982) and (iii) two $3 \rightarrow 1$ hydrogen bonded $\gamma \gamma$-type (Ramakrishnan and Rao 1980). Using grid search technique, several stereochemically satisfactory conformations have been worked out The grid search technique has a lacuna in that the bond lengths and bond angles of the molecule are kept rigid while arriving at the possible minimum energy conformations - A study has therefore, been undertaken to carry out flexible geometry energy minimization on the CPP conformations. The results show that the backbone can adopt several stereochemically good and energetically favourable hydrogen bonded conformations (a preliminary account of results has been published in Nagarajaram and Ramakrishnan 1991).

\section{Method}

\subsection{Generation of conformations}

For generating different conformations of cyclic peptides, the first step is to get a satisfactory ring closure. The procedure used for cyclization developed and used extensively by this group has been, to link two or more peptide fragments. The procedural details as used for the different cases of small cyclic peptides have been described earlier (Manjula and Ramakrishnan 1979; Ramakrishnan and Rao 1980; Paul and Ramakrishnan 1987; Ramakrishnan et al 1987) In the present study too a similar procedure has been used, in which CPP is generated by linking a tripeptide fragment to a dipeptide fragment Figure 1 shows a schematic representation of the CPP as a linked system of tri- and dipeptide fragments. The different steps involved are briefly given below.

(i) Generation of tripeptide fragment by specifying $\left(\phi_{2}, \Psi_{2}\right)$ and $\left(\phi_{3}, \Psi_{3}\right) \cdot$

(ii) Generation of an appropriate dipeptide fragment by specifying the value of $\phi_{5}$ and the end to end distance as that of the tripeptide fragment*.

(iii) Linking of the dipeptide fragment to the tripeptide fragment and tilting it about the virtual bond. $\mathrm{C}_{1}^{\alpha} \ldots . \mathrm{C}_{4}^{\alpha}$ (see figure 1) till the bond angles at the linking $\alpha$-carbon atoms $C_{1}^{\alpha}$ and $C_{4}^{\alpha}$ viz., $\tau_{1}$ and $\tau_{4}$ take up acceptable values (i.e., close to the tetrahedral value $=109 \cdot 5^{\circ}$ )

Using the above procedure satisfactory ring closures can be achieved just by specifying ranges for the independent parameters , $\phi_{2}, \Psi_{2}, \phi_{3}, \Psi_{3}, \phi_{5}$, and the tilt angle. In the present study since the focus is on conformations of CPP with

\footnotetext{
*A dipeptide fragment whose end to end distance equals that of the tripeptide fragment can be generated by specifying appropriate values of $\left(\phi_{5}{ }^{\prime} \psi_{5}\right)$. In the present study, an analytical procedure (Manjula 1976) has been used to compute the values of $\psi_{5}$ for given values of $\phi_{5}$ such that the end to end distance of the dipeptide fragment is same as that of the tripeptide fragment ( $\mathrm{i} \cdot \mathrm{e} \cdot$, the distance $\mathrm{C}_{1}^{\alpha}$ to $\mathrm{C}_{4}^{\alpha} \mathrm{Using}$ the values of $\phi_{5}$ and the corresponding values of $\Psi_{5}$, the dipeptide fragment is generated.
} 


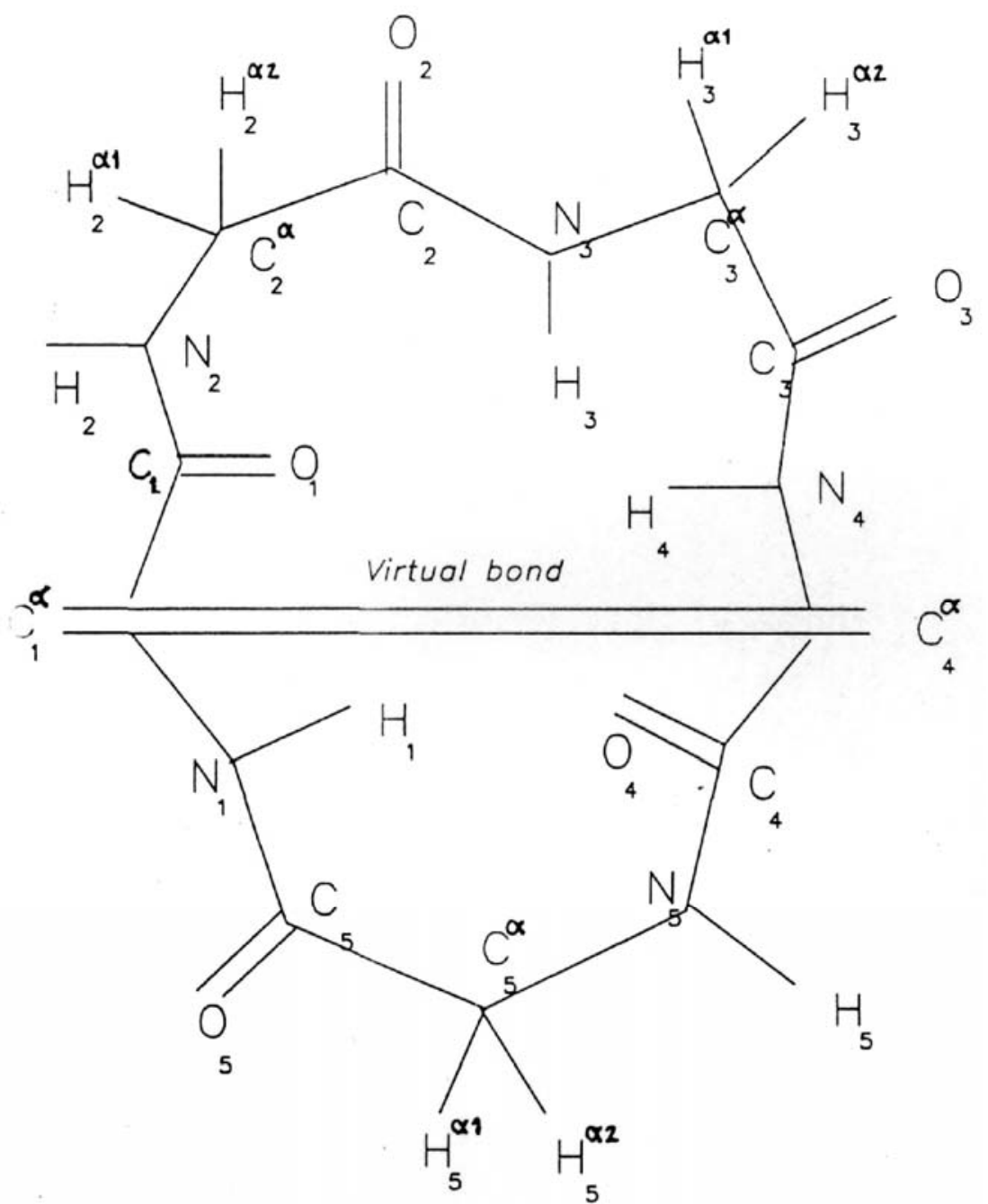

Figure 1- Schematic diagram illustrating the cyclic pentapeptide as a system made up of two linked peptide fragments viz, tripeptide fragment $\left(\mathrm{C}_{1}^{\alpha}\right.$ to $\left.\mathrm{C}_{4}^{\alpha}\right)$ and dipeptide fragment $\left(C_{1}^{\alpha}\right.$ to $C_{4}^{\alpha}$ via $\left.C_{5}^{\alpha}\right)$. Also shown the virtual bond joining $C_{1}^{\alpha}$ and $C_{4}^{\alpha}$

intracyclic hydrogen bonds, the ranges of the above mentioned independent parameters have been chosen such that the generated CPP have intracyclic hydrogen bonds. These ranges are given in table 1. The tilt angle has been varied through the entire range of $0-360^{\circ}$ at suitable intervals.

Among the different residues glycyl has been used at all the $\mathrm{C}^{\alpha^{\prime}}$ s (leading to cyclo pentaglycyl). This has intentionally been done to ensure that none of the possible backbone conformations are eliminated due to backbone-sidechain steric factor- A large number of conformations so generated are subjected to energy 
Table1. The ranges of the independent parameters $\phi_{2} \Psi_{2^{\prime}} \phi_{3^{\prime}} \Psi_{3}$ and $\phi 5$ used in grid search for generation of different hydrogen bonded conformations of CPP.

\begin{tabular}{|c|c|c|}
\hline $\begin{array}{l}\text { Peptide } \\
\text { fragment }\end{array}$ & Conformation & $\begin{array}{l}\text { Range of }(\phi, \psi)^{\prime} s \\
\left({ }^{\circ}\right)\end{array}$ \\
\hline \multirow[t]{6}{*}{ Tripeptide } & $\beta$-turn type II & $\begin{aligned}-100 \leq \phi_{2} \leq-20 ; & 70 \leq \Psi_{2} \leq 160 \\
30 \leq \phi_{3} \leq 170 ; & 70 \leq \Psi_{3} \leq 80\end{aligned}$ \\
\hline & $\beta$-turn type I & $\begin{array}{rc}-80 \leq \phi_{2} \leq-20 ; & -90 \leq \psi_{2} \leq-10 \\
-150 \leq \phi_{3} \leq-70 ; & 20 \leq \psi_{3} \leq 80\end{array}$ \\
\hline & $\gamma$-turn at $C_{2}^{\alpha}$ & $20 \leq \phi_{2} \leq 120 ;-120 \leq \psi_{2} \leq-20$ \\
\hline & $\gamma$-turn at $C_{3}^{\alpha}$ & $20 \leq \phi_{3} \leq 120 ;-120 \leq \psi_{3} \leq-20$ \\
\hline & $\gamma^{\prime}$-turn at $\mathrm{C}_{2}^{\alpha}$ & $-120 \leq \phi_{2} \leq-20 ; \quad 20 \leq \psi_{2} \leq 120$ \\
\hline & $\gamma^{\prime}$-turn at $C_{3}^{\alpha}$ & $-120 \leq \phi_{3} \leq-20 ; \quad 20 \leq \psi_{3} \leq 120$ \\
\hline \multirow[t]{4}{*}{ Dipeptide } & $\gamma$-turn at $C_{5}^{\alpha}$ & $20 \leq \phi_{5} \leq 120 ;-120 \leq \psi_{s} \leq-20$ \\
\hline & $\gamma^{\prime}$-turn at $C_{5}^{\alpha}$ & $-120 \leq \phi_{5} \leq-20 ; \quad 20 \leq \psi_{5} \leq 120$ \\
\hline & Right handed $\alpha$-helical & $-120 \leq \phi_{s} \leq-10 ;-120 \leq \psi_{s} \leq-10$ \\
\hline & Left handed $\alpha$-helical & $10 \leq \phi_{5} \leq 120 ; \quad 10 \leq \psi_{5} \leq 120$ \\
\hline
\end{tabular}

minimization and the host of minima so obtained represent a 'database' of CPP stable conformations which can be considered as prototypes.

The method of energy minimization is same as the one used in the earlier studies by this group (Paul and Ramakrishnan 1985, 1987; Ramakrishnan et al 1987; Nagarajaram et al 1992). The minimization method is based upon the gradient techniques viz; steepest descent technique and Davidon-Fletcher-Powel method (DFP) (Davidon 1959; Fletcher and Powell 1963). The conformations are first subjected to a few cycles of steepest descent (about 90 cycles) and further minimization has been carried out using DFP. The total energy of the system is evaluated as the sum of the contributions from the non-bonded, electrostatic, hydrogen bonding interactions and strain energies arising out of the distortions of the bond lengths and bond angles. Various constants and the forms of potential functions used are same as those given in Ramachandran and Sasisekharan (1968), except for the hydrogen bonding interaction for which the potential function proposed by Balasubramanian et al (1970) has been used.

\subsection{Nomenclature}

During the course of this study, several energy minima have been obtained- Thus, it has become imperative to properly designate each of these minima so that they can be represented properly and distinguished from one another- Since all the energy minima possess intramolecular hydrogen bonds, it was felt that the designation should involve notations based on these hydrogen bonds indicative of associated turn features In view of the importance of proper designation, the underlying principles and the procedural details of the proposed system of designation are given below.

Since CPP can be thought of as a system of linked tripeptide and dipeptide fragments, any hydrogen bonding pattern (and, thus, the basic backbone conformation) in a given conformation can be represented as an assembly of the corresponding turn types. These turns can occur (i) entirely in the tripeptide fragment, (ii) entirely in the dipeptide fragment and (iii) spanning across the tripeptide and dipeptide fragments. 
The designation of any energy minimum can be composed as,

$\left[\begin{array}{c}\text { Hydrogen } \\ \text { bonds in } \\ \text { tripeptide } \\ \text { fragment }\end{array}\right] \begin{gathered}\text { Hydrogen } \\ \text { bonds } \\ \text { flanking } \\ \mathrm{C}_{4}^{\alpha}\end{gathered}\left(\begin{array}{c}\text { Hydrogen } \\ \text { bonds in } \\ \text { dipeptide } \\ \text { fragment }\end{array}\right) \begin{gathered}\text { Hydrogen } \\ \text { bonds } \\ \text { flanking } \\ \mathrm{C}_{1}^{\alpha}\end{gathered}$

in which the hydrogen bonds occurring in the tripeptide fragment are enclosed in square brackets and those occurring in the dipeptide fragment are enclosed in normal parentheses.

The intramolecular hydrogen bonds in all the energy minima obtained in the present study correspond to $\beta$ - and $\gamma$-turns. The usual notations have been used, for representing them [ $\beta^{x}$ - where $X$ is one of the four types I, I', II and II' while for $\gamma$-turns occurring around $\left(80^{\circ},-70^{\circ}\right)$ and $\left(-80^{\circ}, 70^{\circ}\right)$ as $\gamma$ and $\gamma^{\prime}$ respectively]. In order to further specify the location of $\beta$ (or $\gamma$ ) turns, the sequence number of the central $\mathrm{C}^{\alpha}$ atoms contained in the corresponding turn segments are written as subscripts to the yurn notations. For example., $\mathrm{B}_{2.3}^{\mathrm{II}}$ will mean that there is a type II $\beta$-turn encompassing $\mathrm{C}_{2}^{\alpha}$ and $\mathrm{C}_{3}^{\alpha}$

\section{Results and discussion}

Grid search yielded a large number of geometrically permissible conformations and energy minimizations using these as the starting conformations resulted in 23 energy minima [minimum energy conformations (MECs)] Interestingly, all these minima possess intracyclic hydrogen bonds. The hydrogen bond parameters viz; length $(l)$ $\mathrm{N}$. . . O and angle $(\theta) \mathrm{H}-\mathrm{N}$. . O in all the cases lie within the ranges 2.6 to $3.3 \AA$ for $l$ and less than $30^{\circ}$ for.$\theta$

\section{$3 \cdot 1$ Description of MECs}

The hydrogen bonding pattern, turn types and the conformational parameters in the MECs are given in table 2. The hydrogen bonds present in these MECs are of two types (i) $\beta$ and $\gamma$ and (ii) only $\gamma$. Based on the type of the hydrogen bond within the tripeptide fragment, all the MECs are grouped into 4 categories and they are:

Category 1: Tripeptide fragment characterized by $4 \rightarrow 1$ hydrogen bond of type II $\beta$-turn (MECs 1 to 13 in table 2).

Category 2: Tripeptide fragment characterized by $4 \rightarrow 1$ hydrogen bond of type I $\beta$-turn (MECs 14 to 17 in table 2).

\footnotetext{
*In the above method of designation there is no ambiguity if there are hydrogen bonds in both the tripeptide and dipeptide fragments as well as across the linking sites $\left(\mathrm{C}_{1}^{\alpha}\right.$ and $\left.\mathrm{C}_{4}^{\alpha}\right)$. However, it is possible that for some minima some of these hydrogen bonds are absent. In such situations the symbol hyphen ("-") is used at the relevant places and the designation is composed For example, in a case where there are no hydrogen bonds within the dipeptide fragment as well as across $\mathrm{C}_{4}^{\alpha}$ but there are hydrogen bonds of type II $\beta$-turn within the tripeptide fragment and a $\gamma$-turn across $C_{1}^{\alpha}$ the designation would be $\left[\beta_{2,3}^{11}\right]-(-) \gamma_{1}$.
} 
Table 2- Hydrogen bonding pattern and the conformational parameters of MECs. The nonplanarity of the five peptide units represented by $\Delta \omega\left(\Delta \omega=180^{\circ}-\omega\right)$ is given, in parentheses, below the values of $(\phi, \psi)$.

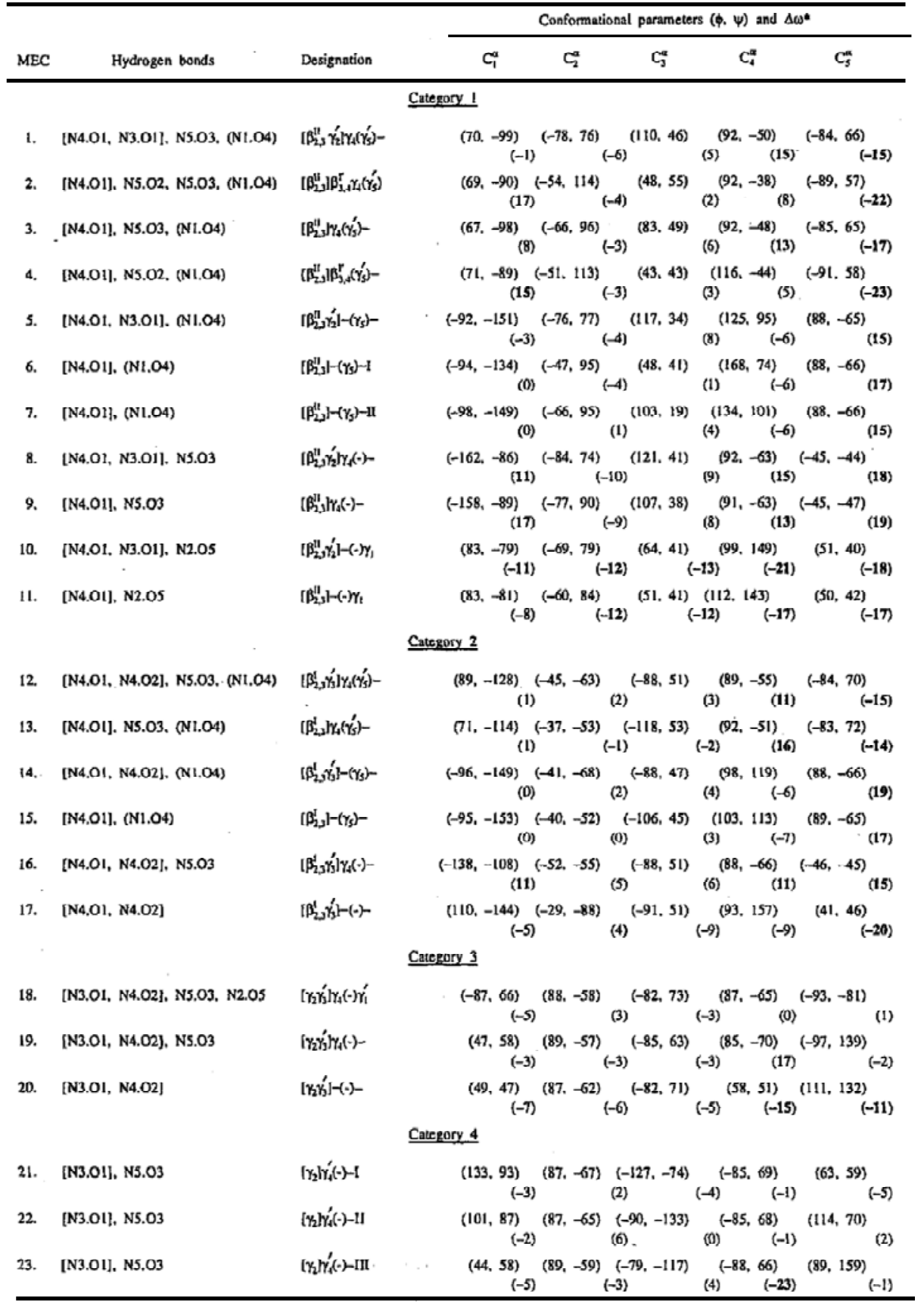

*Values of $\Delta \omega>10$ are given as bold. 
Category 3: Tripeptide fragment characterized by a pair of contiguous $3 \rightarrow 1$ hydrogen bonds of $\gamma$-turns (MECs 18 to 20 in table 2).

Category 4: Tripeptide fragment characterized by a single $3 \rightarrow 1$ hydrogen bond of $\gamma$-turn (the last three entries of table 2).

In the following sections the salient features of the MECs belonging to each category are described.

\section{(i) Category 1}

This category is constituted by 11 MECs (MEC1 to MECll). This shows that nearly half of the number of MECs obtained in this study belong to this category.

Of the 11 MECs there are 4 examples wherein the tripeptide fragment has also accommodated $\gamma^{\prime}$-turn that coexists with the $\beta$-turn. This has resulted in the formation of a system of hydrogen bonds sharing the common acceptor atom. The dipeptide fragment has assumed 4 different conformations two of which are the well- known $\gamma$ and $\gamma^{\prime}$-turns with the characteristic $3 \rightarrow 1$ hydrogen bonds and the other two are non-hydrogen bonded. The conformation of the dipeptide fragment in the latter two cases either corresponds to the right handed $\alpha$-helical $\left(\alpha_{R}\right)$ or left handed $\alpha$-helical $\left(\alpha_{L}\right)$ region of the $(\phi, \Psi)$-map.

Apart from the hydrogen bonds of intrafragmental nature, there are also hydrogen bonds of the types $3 \rightarrow 1$ and $4 \rightarrow 1$ which have formed between the fragments. Their formations has been made possible due to the characteristic conformations assumed by the linking sites.

Thus, in this category of MECs the noteworthy aspect is that, though the conformation of the tripeptide fragment is nearly fixed as type II $\beta$-turn, the conformational variety among the MECs arises due to the different conformations assumed by the dipeptide fragment and the two linking sites.

The line diagrams and the corresponding projection diagrams for these 11 MECs are shown in figure 2 .

(ii) Category 2

This category constitutes 6 MECs. In 4 of these, the hydrogen bonds in tripeptide fragment are bifurcated in nature, formed by the coexistence of type I $\beta$ - and $\gamma^{\prime}$-turns.

As in the earlier case, in this category too, one can see conformational variety exhibited by the dipeptide fragment and the two linking sites. However, the number of MECs in this category are less compared to the earlier category. This means that the CPP backbone has less flexibility when it adopts type I $\beta$-turn than when it adopts type $11 \beta$-turn, though, the dipeptide fragment and the linking sites can adopt similar conformations.

Figure 3 shows the line diagrams and the projection diagrams of the MECs of this category.

(iii) Categories 3 and 4

In both these categories of MECs the backbone possesses only $\gamma$-type hydrogen bonds.The chief difference between the MECs belonging to these two categories 
(a)
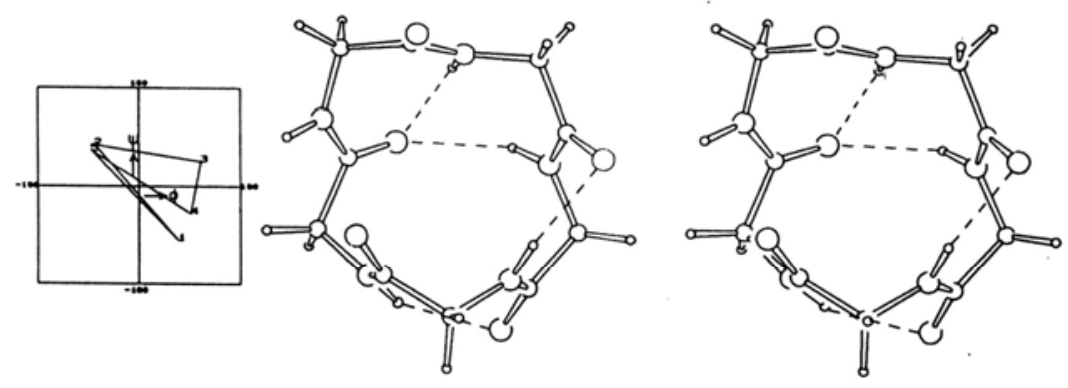

(b)
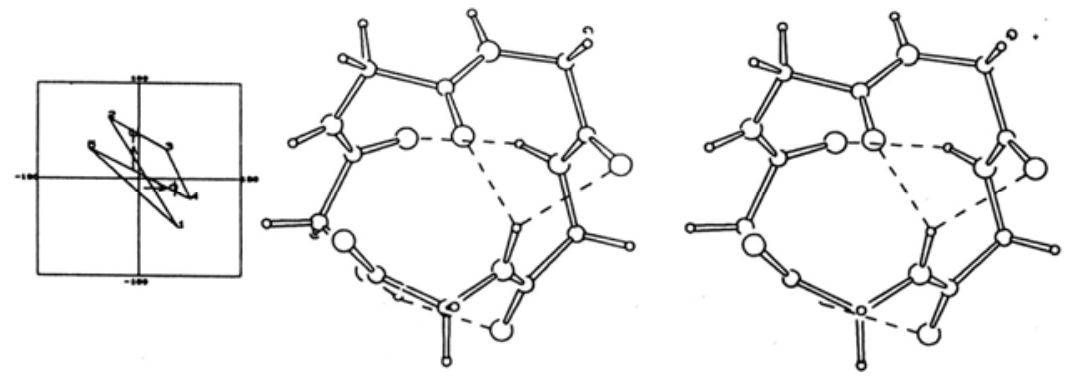

(c)
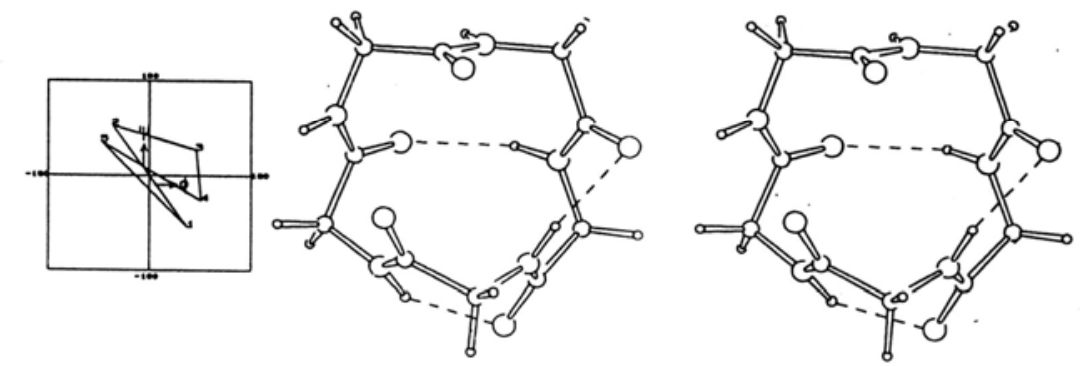

(d)
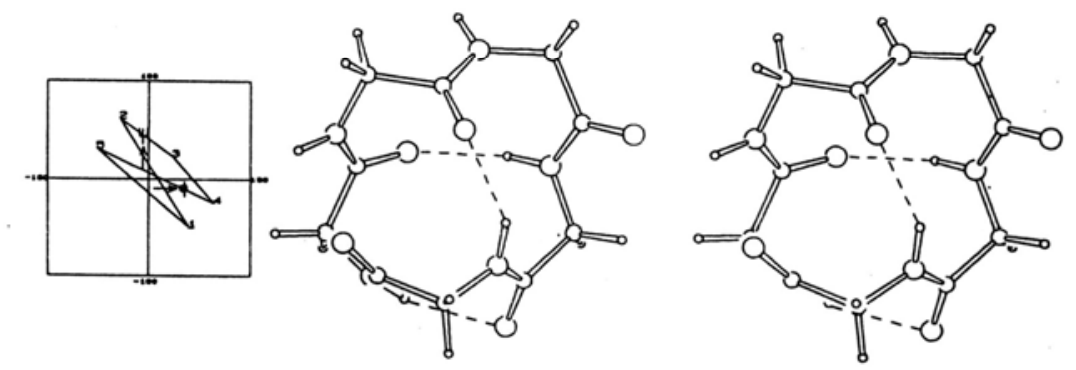

Figure 2a-d. 
(e)
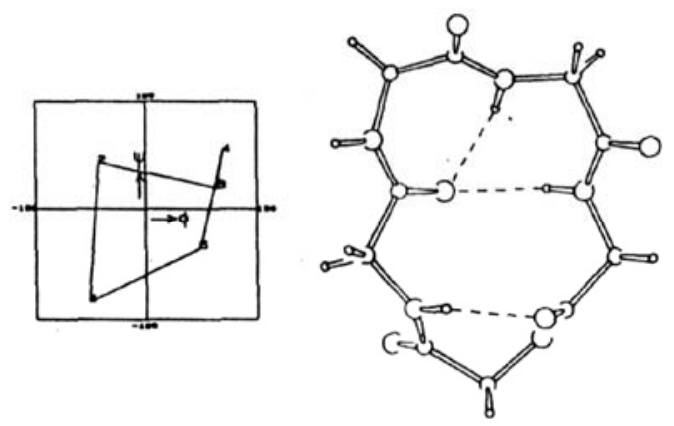

(f)
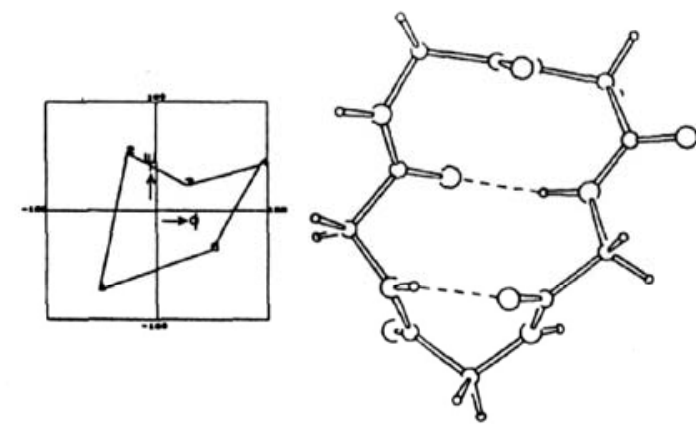

(g)
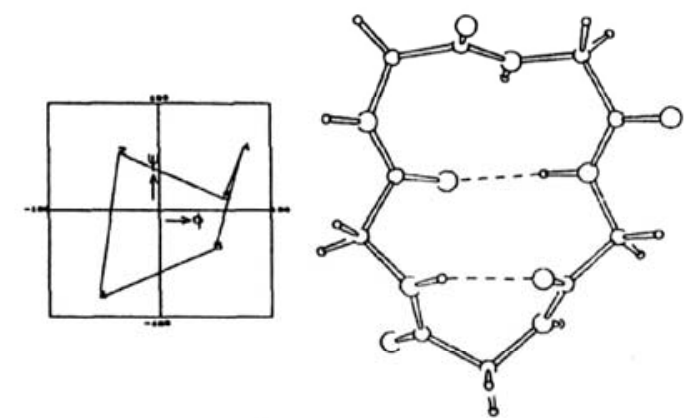

(h)
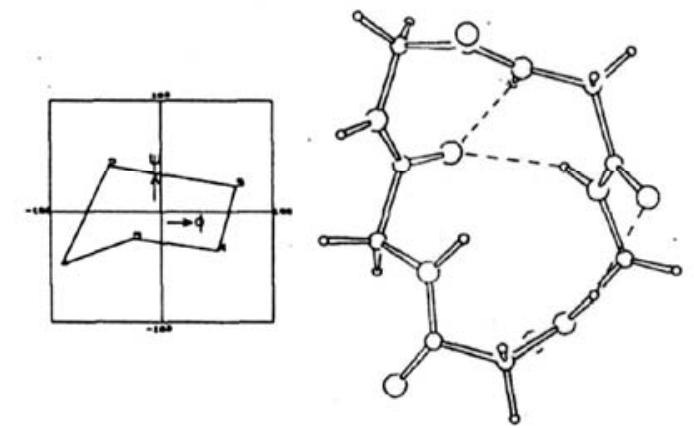
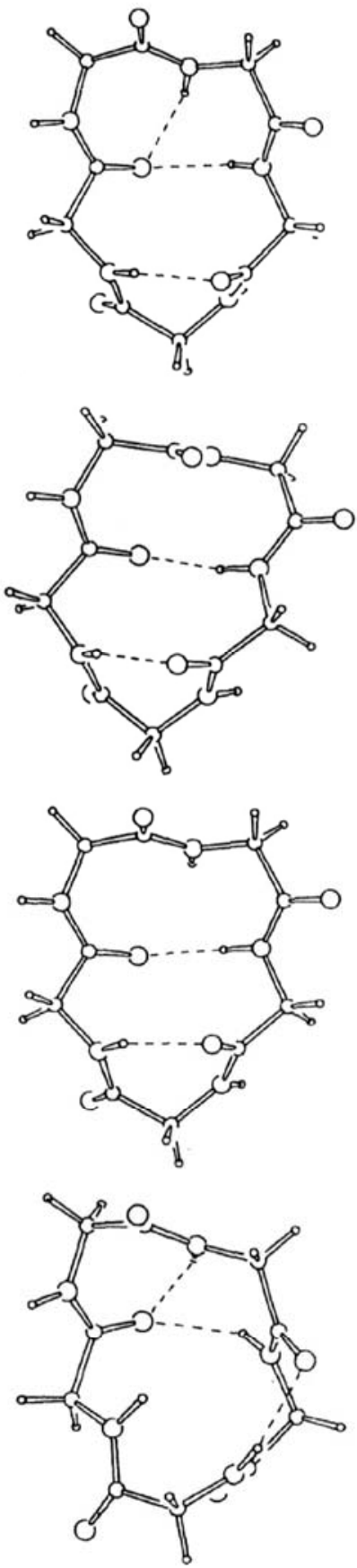

Figure 2e-h. 
(i)
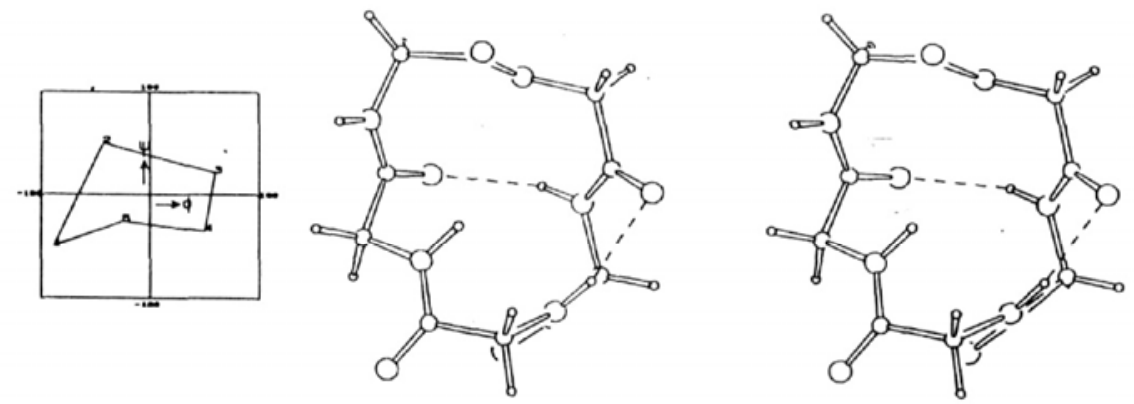

(j)
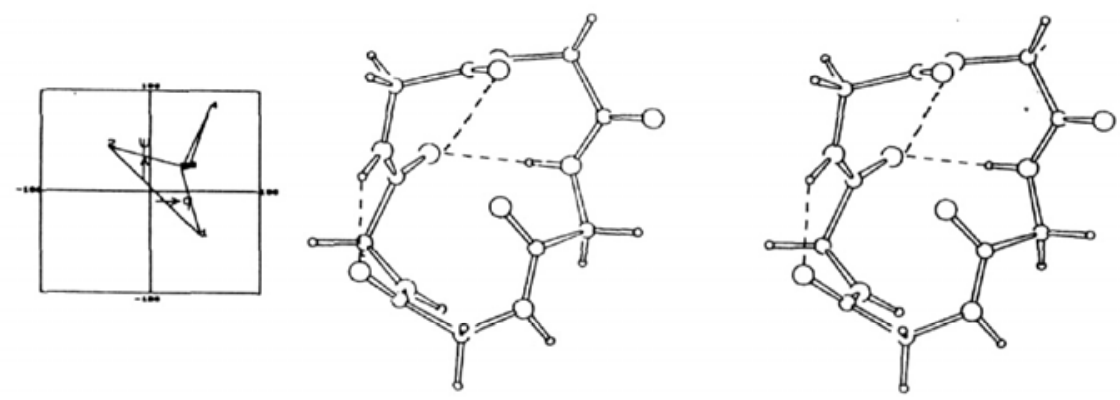

$(k)$
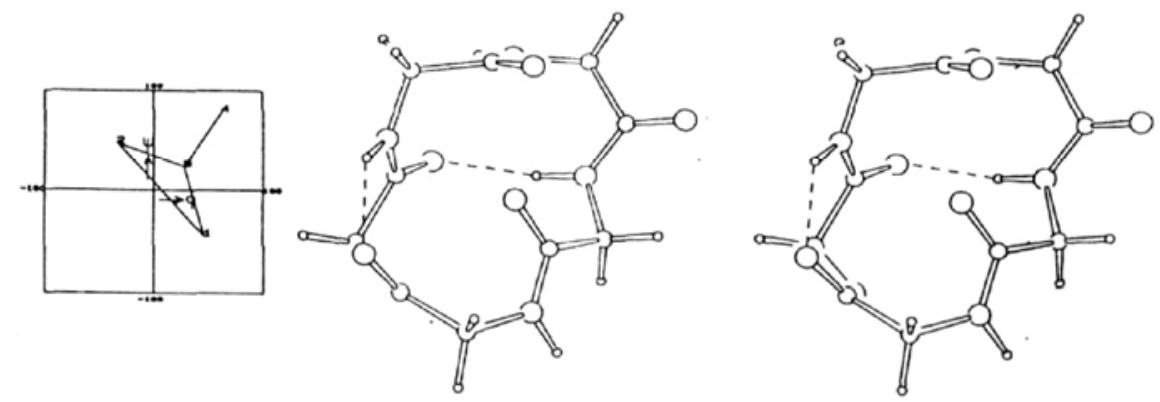

Figure 2. The line diagrams and the corresponding projection diagrams (stereo pairs) of the 11 MECs (MEG1 to MEC11) belonging to category 1 . The hydrogen bonds are shown as broken lines.

is the number of $\gamma$-turns formed within the tripeptide fragment- In category 3 two contiguous $\gamma$-turns are formed within the tripeptide fragment whereas in category 4 only one $\gamma$-turn is formed. The line-diagrams along with their projection diagrams are shown in figure 4 and 5 .

The chief differences among the MECs of category 3 are the number and location of additional hydrogen bonds that occur in them. In all the cases the dipeptide fragment is devoid of a hydrogen bond- One of the interesting result is in MEC18, in which both the linking $\alpha$-carbon atoms viz, $C_{1}^{\alpha}$ and $C_{4}^{\alpha}$ are flanked by $3 \rightarrow 1$ type hydrogen bonds, belonging to inverse $\gamma$ and $\gamma$-turns respectively. The consequence of this is that the backbone has four contiguous hydrogen bonds involving four out of five $\mathrm{N}-\mathrm{H}$ and $\mathrm{C}=\mathrm{O}$ groups available for hydrogen bonding. 
(a)
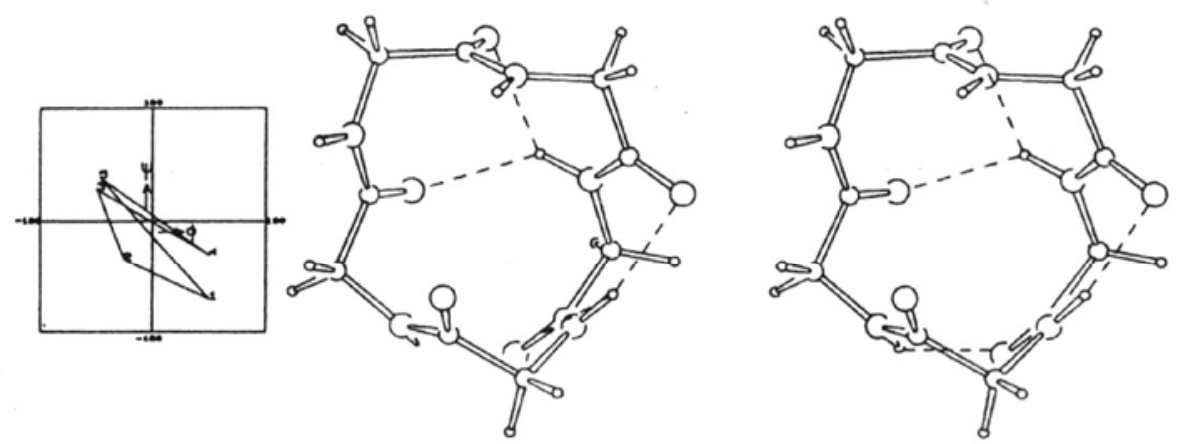

(b)
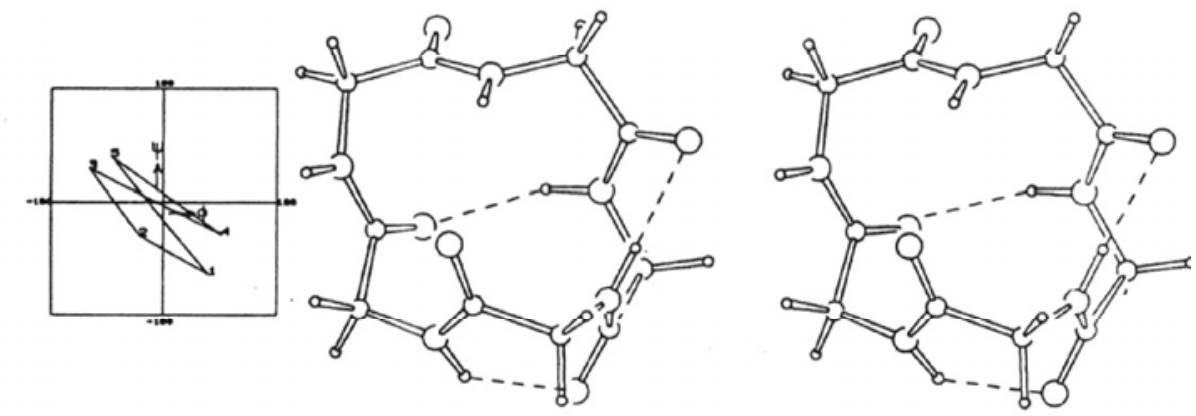

(C)
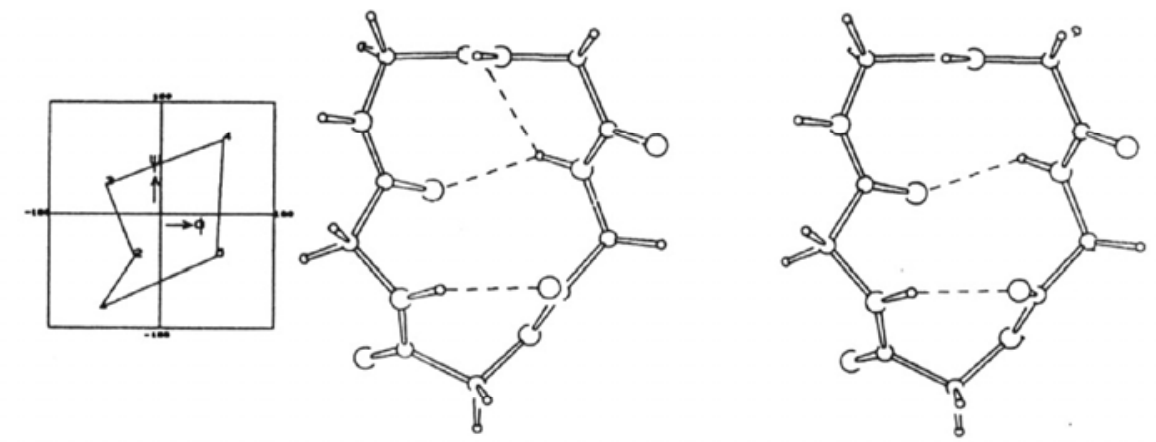

(d)
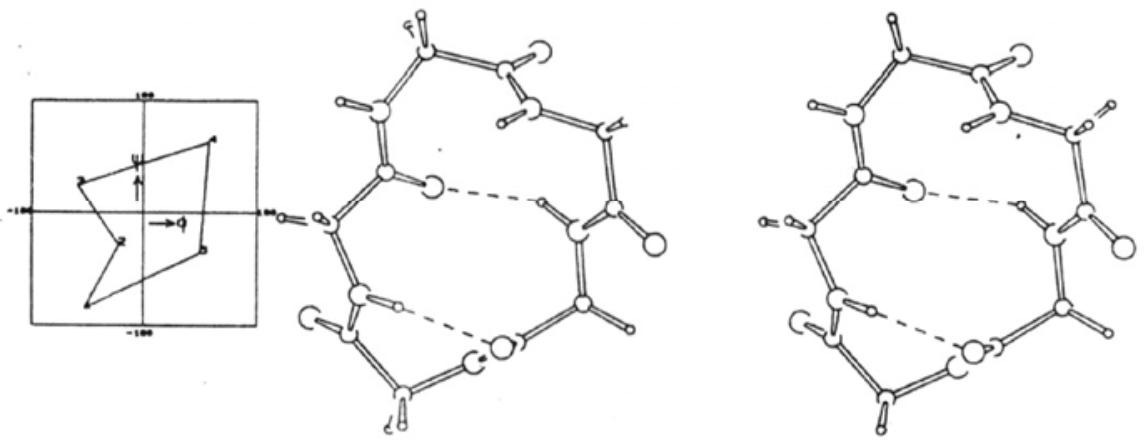

Figure 3a-d 
(e)
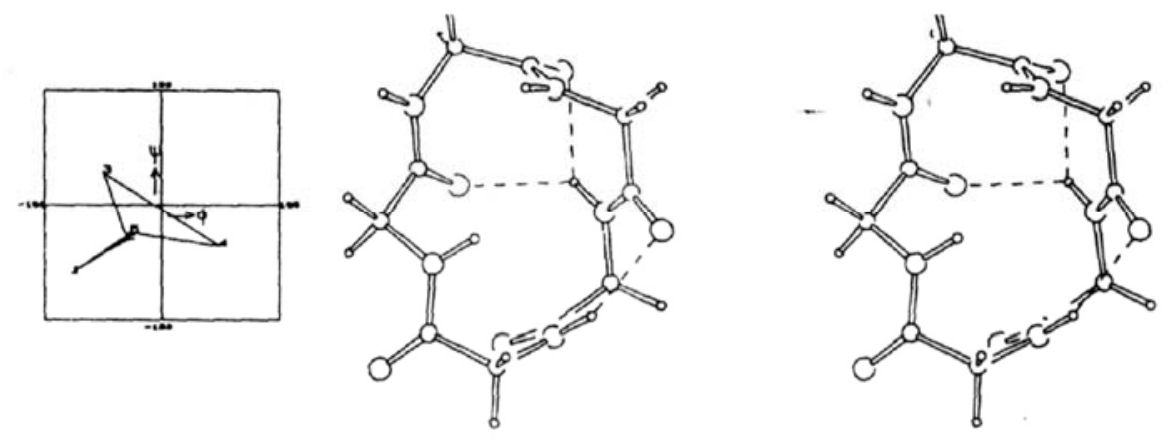

(f)
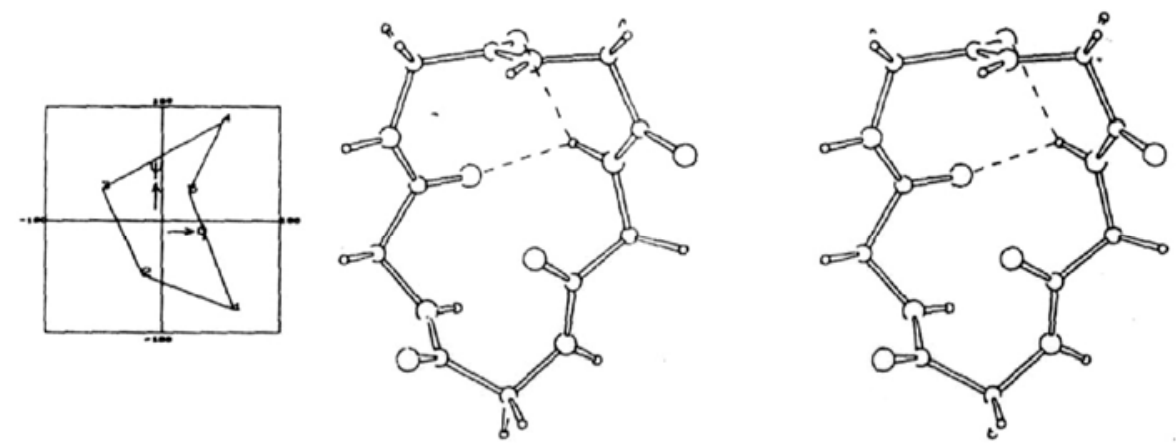

Figure 3. The line diagrams and the corresponding projection diagrams (stereo pairs) of the 6 MECs (MEC12 to MEC17) belonging to category 2. The hydrogen bonds are shown as broken lines.

In category 4 all the three MECs (21 to 23) have the same pattern of hydrogen bonds. However, they are very distinct from the conformational view point This is well reflected by the different $(\varphi, \psi)$ angles at $C_{1}^{\alpha}, C_{3}^{\alpha}$ and $C_{5}^{\alpha}$ as given in table 2 .

\subsection{Planarity of the peptide units}

Earlier studies on CPP have shown that it is possible for CPP to adopt conformations in which the peptide units are strictly planar (Ramakrishnan and Sarathy 1969; Manjula 1976; Ramakrishnan and Rao 1980; Rao 1982). However, to form some specific structural types (such as $\beta, \gamma$-structures) it has been found that at least one peptide unit needs to be distorted from planarity (Rao 1982). The non-planarity of the peptide units is a common feature in all the CPPs studied in the solid state by X-ray crystallography (Karle 1978, 1979, 1981, 1986; Mauger et al 1982; Stradley et al 1990). In what follows, this important conformational aspect has been examined vis-a-vis, the hydrogen bonding pattern and associated turn types.

In table 2 the amount of non-planar distortion for the five peptide units quantified by the parameter $\Delta \omega(\Delta \omega=180 \cdot 0-\omega)$ are given. It can be seen that non-planarity has become an inherent feature for most of the MHCs. The amount of non-planarity goes up as high as $20^{\circ}$ in some of the cases. 
(a)
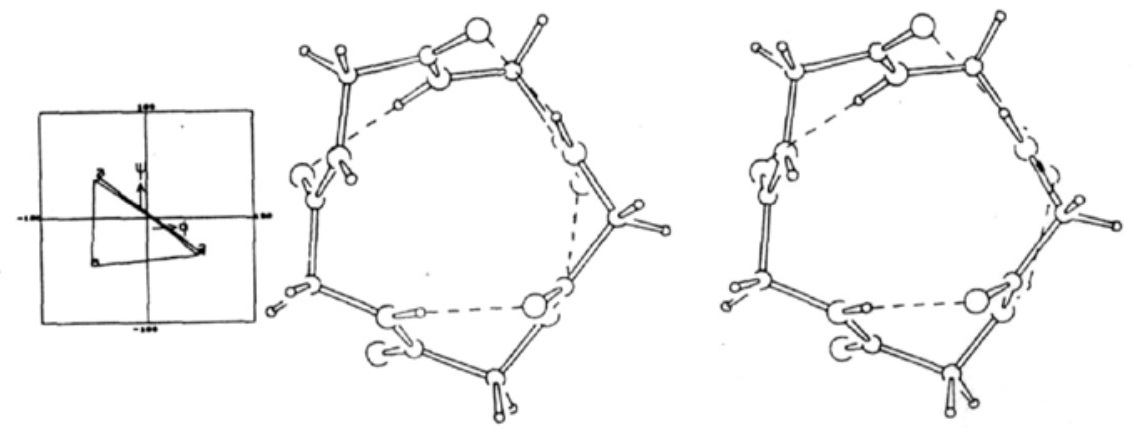

(b)
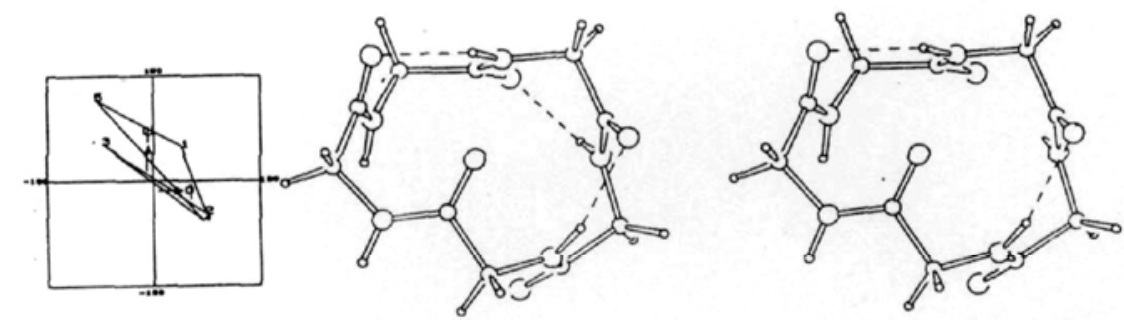

(C)
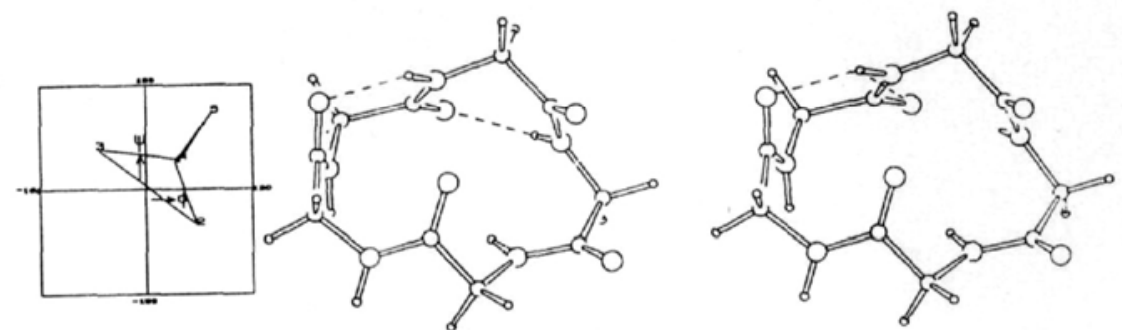

Figure 4. The line diagrams and the corresponding projection diagrams (stereo pairs) of the 3 MECs (MEC 18 to MEC20) belonging to category 3. The hydrogen bonds are shown as broken lines.

By considering any value of $|\Delta \omega|$ grater than $10^{\circ}$ as indicative of significant measure of non-planarity for a peptide unit, the following aspects can be perceived from table 2 .

(i) Majority of the MECs (20 MECs out of 23) possess at least one significantly non-planar peptide unit- In MEC10 all the peptide units exhibit significant deviation from planarity. In MECs 8 and 11, four out of five units show significant deviation. (ii) In all the MECs having $\beta$-turn in the tripeptide fragment $(i \cdot e \cdot$, those belonging to the categories 1 and 2) the non-planar deviation is mostly confined to the dipeptide fragment $i \cdot e \cdot$, between $C_{4}^{\alpha}$ to $C_{1}^{\alpha}$ via $C_{5}^{\alpha}$. Among these the fifth peptide unit (between $\mathrm{C}_{5}^{\alpha}$ to $\mathrm{C}_{1}^{\alpha}$ ) exhibits significant non-planarity. 

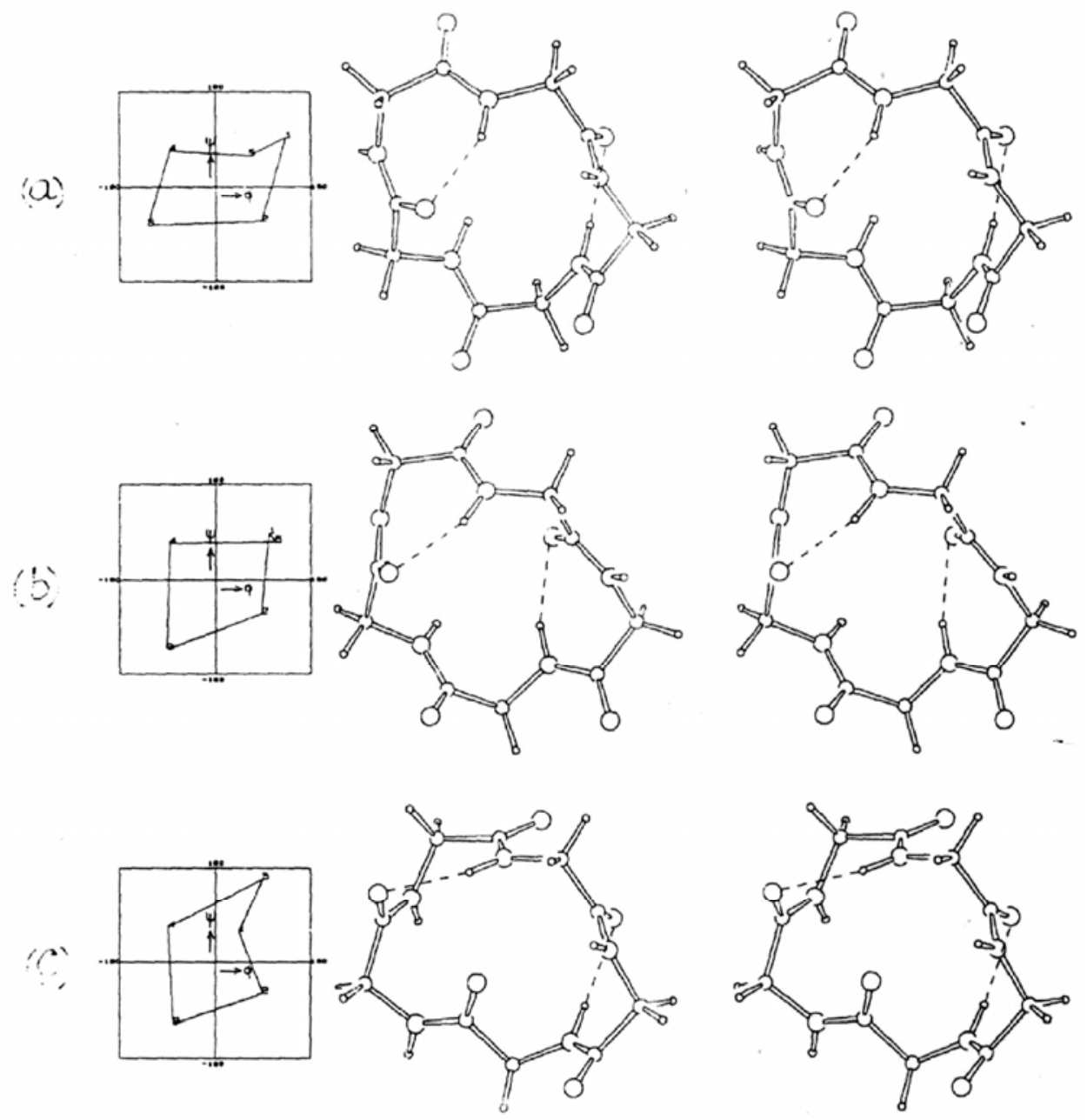

Figure 5. The line diagrams and the corresponding projection diagrams (stereo pairs) of the 3 MECs (MEC21 to MEC23) belonging to category 4. The hydrogen bonds are shown as broken lines.

(iii) In those cases where the significant non-planarity is exhibited by more than one peptide unit, the peptide units involved are adjacent (e.g., $\Delta \omega_{4}$ and $\left.\Delta \omega 5\right)$ in MEC1 and $\Delta \omega_{5}$ and $\Delta \omega_{1}$ in MEC2).

(iv) The number of peptide units that are significantly non-planar are less in MECs which have only $\gamma$-turns than those which have $\beta$ - and $\gamma$-turns in them. The consistent occurrence of a significant non-planar peptide unit in the dipeptide fragment of the MECs which have $\beta$-turn in its tripeptide fragment will probably mean that, occurrence of a ß-turn in a CPP has a geometrical requirement for non-planar distortion. 
Energetically, non-planar distortion and hydrogen bonding have opposite effects on the stability of conformation (the former reducing the stability and the latter increasing it) and the fact that, a large number of energy minima with hydrogen bonds have been obtained in the minimization process indicates that the stabilizing effect of hydrogen bond is the dominating factor in spite of the destabilizing effect of non-planar distortion.

\subsection{Conformational families}

It is clear that each MEC is characterized by a unique pattern of hydrogen bonds. However, a look at either the angles or the line diagrams reveals that not all of them are conformationally totally distinct and that some of the line diagrams have similar shapes indicating probable conformational similarity among the corresponding MECs. This group of MECs which have similar $\phi, \Psi$ 's forms a cluster in the conformational space (a 10-dimensional space spanned by the 10 parameters $\phi_{1}, \Psi_{1} \ldots \ldots ., \phi_{5}$ and $\left.\Psi_{5}\right)$ though they are characterized by different patterns of hydrogen bonds. Such a cluster of MECs in the conformational space is henceforth referred to as a "conformational family".

While searching for the MECs that can form a conformational family, it is essential to keep in mind the following aspects.

(i) For every MEC there is an inverse MEC with all $(\phi, \Psi)$ 's inverted Such an inverse MEC will have the same hydrogen bonding characteristics as that of the parent MEC.

(ii) Cyclic permutation of the $(\phi, \Psi)$ angles at the five $\alpha$-carbon atoms must be carried out when testing for similarity with another MEC.

Since subjective element is to be avoided, the following quantitative method has been employed to identify the groups of MECs such that within each group the members are close to one another in the 10-dimensional conformational space.

3.3a Distance method of finding conformational families: Any MEC can be represented as a point in the 10-dimensional space with the 5 pairs of $(\phi-\Psi)$ angles constituting the 10 parameters . It is quite easily perceptible that, in the case of MECs which are in the same region of the conformational space (thus having similar conformational angles), "the 10-dimensional distance" of separation will be much smaller compared to the distances of separation of those MECs which are in different regions of the conformational space (having dissimilar conformational angles). Thus the 10-dimensional distance can be used as an useful quantity to find out the clusters of the MECs.

The 10-dimensional distance, denoted by $d_{i j}$, between the MEC $i$ and MEC $j$ is evaluated as:

$$
d_{i j}=\sqrt{\left(\Delta \phi_{1}^{2}+\Delta \psi_{1}^{2}+\ldots+\Delta \phi_{s}^{2}+\Delta \psi_{s}^{2}\right)}
$$

where, etc $, \Delta \phi_{1}, \Delta \psi_{1} *$ are the differences between the values of the corresponding conformational angles $\phi_{1}, \Psi_{1}$, etc, of the two MECs $i$ and $j$. While calculating the 10-dimensional distance between $i$ th and $j$ th MECs by using the above expression,

*Care has been taken to consider the least value of the differences in the values of the $\phi$ 's. and $\psi$ 's For example, if $\phi_{i}=160^{\circ}$ and $\phi_{j}=-140^{\circ}$, then the difference is not $300^{\circ}$ but only $60^{\circ}$. 
the five possible values obtained by the cyclic permutation of the $(\varphi, \psi)$ values of the $j$ th MECs have been considered and the smallest of these is taken as the actual measure of the separation distance in the 10-dimensional conformational space-

3.3b Distribution of MECs in various conformational families: The 10-dimensional distances have been calculated among the members in a set comprising of $46 \mathrm{MECs}$ (comprising of 23 MECs and their inverses) and scrutinized.

Table 3. MECs grouped into 5 different conformational families (A to E).

\begin{tabular}{|c|c|c|c|}
\hline MEC & Category & Hydrogen bonds and turn types* & $E_{\mathrm{tox}} * *$ \\
\hline \multicolumn{4}{|c|}{ Family A } \\
\hline 1 & 1 & $\mathrm{~N} 4.01\left(\beta^{\prime \prime}\right), \mathrm{N} 3.01\left(\gamma^{\prime}\right), \mathrm{N} 5.03(\gamma), \mathrm{N1.04}\left(\gamma^{\prime}\right)$ & $2 \cdot 8$ \\
\hline 2 & 1 & N4.O1( $\left.\beta^{\prime \prime}\right), N 5.02\left(\beta^{\prime}\right), N 5.03(\gamma)$, N1.04 $\left(\gamma^{\prime}\right)$ & $2 \cdot 9$ \\
\hline 3 & 1 & $\mathrm{~N} 4.01\left(\beta^{\prime \prime}\right), \mathrm{N} 5.03(\gamma), \mathrm{N1.04}\left(\gamma^{\prime}\right)$ & $3 \cdot 5$ \\
\hline 4 & 1 & $\mathrm{~N} 4.01\left(\beta^{\prime \prime}\right), \mathrm{N} 5.02\left(\beta^{\prime}\right), \mathrm{N} 1.04\left(\gamma^{\prime}\right)$ & $2 \cdot 6$ \\
\hline $12^{\prime}$ & 2 & $\mathrm{~N} 5.02\left(\beta^{\prime}\right), \mathrm{N} 5.03(\gamma), \mathrm{N} 1.04\left(\gamma^{\prime}\right), \mathrm{N} 2.05(\gamma)$ & $2 \cdot 3$ \\
\hline $13^{\prime}$ & 2 & $\mathrm{~N} 5 . \mathrm{O} 2\left(\beta^{1^{\prime}}\right), \mathrm{N} 1.04\left(\gamma^{\prime}\right), \mathrm{N} 2 . \mathrm{O} 5(\gamma)$ & $3 \cdot 1$ \\
\hline $18^{\prime}$ & 3 & $\mathrm{~N} 3.01\left(\gamma^{\prime}\right), \mathrm{N} 5.03(\gamma), \mathrm{N} 1.04\left(\gamma^{\prime}\right), \mathrm{N} 2.05(\gamma)$ & 0.0 \\
\hline 19 & 3 & $\mathrm{~N} 5.03(\gamma), \mathrm{N} 1.04\left(\gamma^{\prime}\right), \mathrm{N} 2 . \mathrm{O} 5(\gamma)$ & $2 \cdot 6$ \\
\hline \multicolumn{4}{|c|}{ Family B } \\
\hline 14 & 2 & $\mathrm{~N} 4 . \mathrm{O} 1\left(\beta^{\prime}\right), \mathrm{N} 4 . \mathrm{O} 2\left(\gamma^{\prime}\right), \mathrm{N} 1.04(\gamma)$ & $7 \cdot 1$ \\
\hline 15 & 2 & $\mathrm{~N} 4 . \mathrm{O} 1\left(\beta^{\prime}\right), \mathrm{N1.04}(\gamma)$ & 8.0 \\
\hline $23^{\prime}$ & 4 & $\mathrm{~N} 4.02\left(\gamma^{\prime}\right), \mathrm{N} 1.04(\gamma)$ & $7 \cdot 8$ \\
\hline \multicolumn{4}{|c|}{ Family C } \\
\hline 10 & 1 & $\mathrm{~N} 3.05\left(\beta^{\mathrm{II}}\right), \mathrm{N} 2.05\left(\gamma^{\prime}\right), \mathrm{N} 1.04(\gamma)$ & $8 \cdot 6$ \\
\hline 11 & 1 & $\mathrm{~N} 3.05\left(\beta^{\mathrm{l}}\right), \mathrm{N} 1.04(\gamma)$ & 8.8 \\
\hline $16^{\prime}$ & 2 & $\mathrm{~N} 2.05\left(\gamma^{\prime}\right), \mathrm{N} 1 . \mathrm{O} 3\left(\beta^{\mathrm{r}}\right), \mathrm{N} 1.04(\gamma)$ & $8 \cdot 2$ \\
\hline 20 & 3 & $\mathrm{~N} 2.05\left(\gamma^{\prime}\right), \mathrm{N} 1.04(\gamma)$ & 8.0 \\
\hline \multicolumn{4}{|c|}{ Family D } \\
\hline 5 & 1 & $\mathrm{~N} 4.01\left(\beta^{\prime \prime}\right), \mathrm{N} 3.01\left(\gamma^{\prime}\right), \mathrm{N} 1.04(\gamma)$ & 8.0 \\
\hline 6 & 1 & $\mathrm{~N} 4.01\left(\beta^{\prime \prime}\right), \mathrm{N} 1.04(\gamma)$ & 8.1 \\
\hline 7 & 1 & $\mathrm{~N} 4.01\left(\beta^{\mathrm{II}}\right), \mathrm{N} 1.04(\gamma)$ & $8 \cdot 3$ \\
\hline 22 & 4 & $\mathrm{~N} 3.01\left(\gamma^{\prime}\right), \mathrm{N} 1.04(\gamma)$ & 8.4 \\
\hline \multicolumn{4}{|c|}{ Family E } \\
\hline 8 & 1 & N5.O2 $\left(\beta^{\prime \prime}\right), N 4.02\left(\gamma^{\prime}\right)$, N1.O4 $(\gamma)$ & 9.1 \\
\hline 9 & 1 & N5.02( $\left.\beta^{\prime \prime}\right)$, N1.04 $(\gamma)$ & 9.9 \\
\hline $21^{\prime}$ & 4 & $\mathrm{~N} 4.02\left(\gamma^{\prime}\right), \mathrm{N} 1.04(\gamma)$ & 8.4 \\
\hline
\end{tabular}

*The common hydrogen bond ( $\gamma$-turn N $1 \ldots 04$ ) found in the families is shown as bold. **Total energy is expressed $\mathrm{w} \cdot \mathrm{r} \cdot \mathrm{t} \cdot$ the global energy minimum MEC18 which is taken as $0 \mathrm{kcal} \mathrm{mol}^{-1}$ 
Table 4. Comparison of solid state conformations of the CPPs with the corresponding closest MECs.

\begin{tabular}{|c|c|c|c|c|}
\hline Compound & $\begin{array}{l}\text { Hydrogen bonding } \\
\text { pattern in the } \\
\text { observed example }\end{array}$ & $\begin{array}{c}\text { Closest } \\
\text { MEC }\end{array}$ & $\begin{array}{l}\text { Hydrogen bonding } \\
\text { pattern in the } \\
\text { closest MEC }\end{array}$ & Reference \\
\hline c(Gly-Pro-Gly-D-Ala-Pro) & {$\left[\beta_{2,3}^{\prime \prime}\right]-\left(\gamma^{\prime}\right)-$} & MEC4 & {$\left[\beta_{2,3}^{\prime \prime}\right] \beta_{3,4}^{r}\left(\gamma_{5}^{\prime}\right)-$} & Karle 1978 \\
\hline c(D-Phe-Pro-Gly-D-Ala-Pro) & {$\left[\beta_{2,3}^{\prime \prime}\right]-\left(\gamma^{\prime}\right)-$} & MEC4 & {$\left[\beta_{2,3}^{\mathrm{U}}\right] \beta_{3,4}^{\mathrm{r}}\left(\gamma_{5}^{\prime}\right)-$} & Karle 1981 \\
\hline c(Pro-Gly-Pro-Ser-D-Ala) & {$\left[\beta_{2.3}^{\prime \prime \prime}\right]-(-)-$} & MEC4' & {$\left[\beta_{2,3}^{\mathbb{I I}}\right] \beta_{3,4}^{\prime}\left(\gamma_{5}\right)-$} & Karle 1979 \\
\hline c(Pro-Sar-MeAla-Thr-D-Val) & {$\left[\beta_{2,3}^{\mathrm{II}}\right]-(-)-$} & MEC4' & {$\left[\beta_{2,3}^{\mathrm{II}}\right] \beta_{3,4}^{\prime}\left(\gamma_{5}\right)-$} & Mauger et al 1982 \\
\hline c(Gly-Pro-D-Phe-Gly-Ala) & {$\left[\beta_{2.3}^{\prime \prime}\right]-(-)-$} & MECII & {$\left[\beta_{2,3}^{\prime \prime}\right]-(-) \gamma_{1}$} & Karle 1986 \\
\hline c(Gly-Pro-D-Phe-Gly-Val) & {$\left[\beta_{2,3}^{\prime \prime}\right]-(-)-$} & MEC9 & {$\left[\beta_{2,3}^{\| \prime}\right] \gamma_{4}(-)-$} & Stroup et al 1987 \\
\hline
\end{tabular}

It has been found that, of the $46 \mathrm{MECs}, 44$ are distributed in 10 different conformational clusters. Of these, 5 are distinct families and the remaining 5 are those which can be considered as the "inverse" families. The 5 distinct families are designated by $\mathrm{A}, \mathrm{B}, \mathrm{C}, \mathrm{D} \cdot$ and $\mathrm{E}$ (their inverses by $\mathrm{A}^{\prime}, \mathrm{B}^{\prime}, \mathrm{C}^{\prime}, \mathrm{D}^{\prime}$, and $\mathrm{E}^{\prime}$ ). The families A, B, C, D, and E comprise of 8, 3, 4, 4 and 3 MECs respectively.

The list of MECs constituting different conformational families are given in table 3 The line-diagrams of the members in each of these families are superposed and are shown in figure 6 where the conformational similarity among the members in a family becomes immediately apparent.

The MECs that do not form part of any of the above five families are MEC17 and its inverse MEC17'. MEC 17 forms a separate family designated as F (with only one member) and MEC17' as family $\mathrm{F}^{\prime}$.

3.3c A profile of the conformational space: A conformational family occupies a specific region in the conformational space. Since the MECs are distributed in 12 conformational families, only these many regions of the conformational space are stereochemically as well as energetically favourable for an all-trans CPP.

The global energy minimum in the set of 23 MECs corresponds to MEC18 that has four contiguous $\gamma$-turns. The total energy of the other MECs have been expressed relative to this global energy minimum (which is taken as $0 \mathrm{kcal} \mathrm{mol}^{-1}$ ) and range of the relative total energies in each family is given in column 4 of table 3 .

From the distributions of relative energies in the families, it can be seen that family $\mathrm{A}$ is constituted exclusively of MECs having relatively small energies (which also contains MEC18) while, at the other end of the spectrum lies family $\mathrm{F}$ which constitutes a solitary member MEC17 having relative energy $\approx 11 \mathrm{kcal} \mathrm{mol}^{-1}$.

3.3d Hydrogen bonding pattern in a conformational family: The information pertaining to the intracyclic hydrogen bonds and the category to which each MEC belongs is given in table 3. From this table it can been seen that, every family comprises of MECs of at least two categories. An examination of the families from the hydrogen bonding poinl of view revealed that one of the hydrogen bonds is common among the members of a family. Interestingly the common hydrogen bond is of a $\gamma$ type (see table 3). This means that, in a family, the conformation of the dipeptide segment in the backbone of the MECs is conserved in the form of a $\gamma$-turn while the rest of the backbone possesses a limited flexibility to adopt 

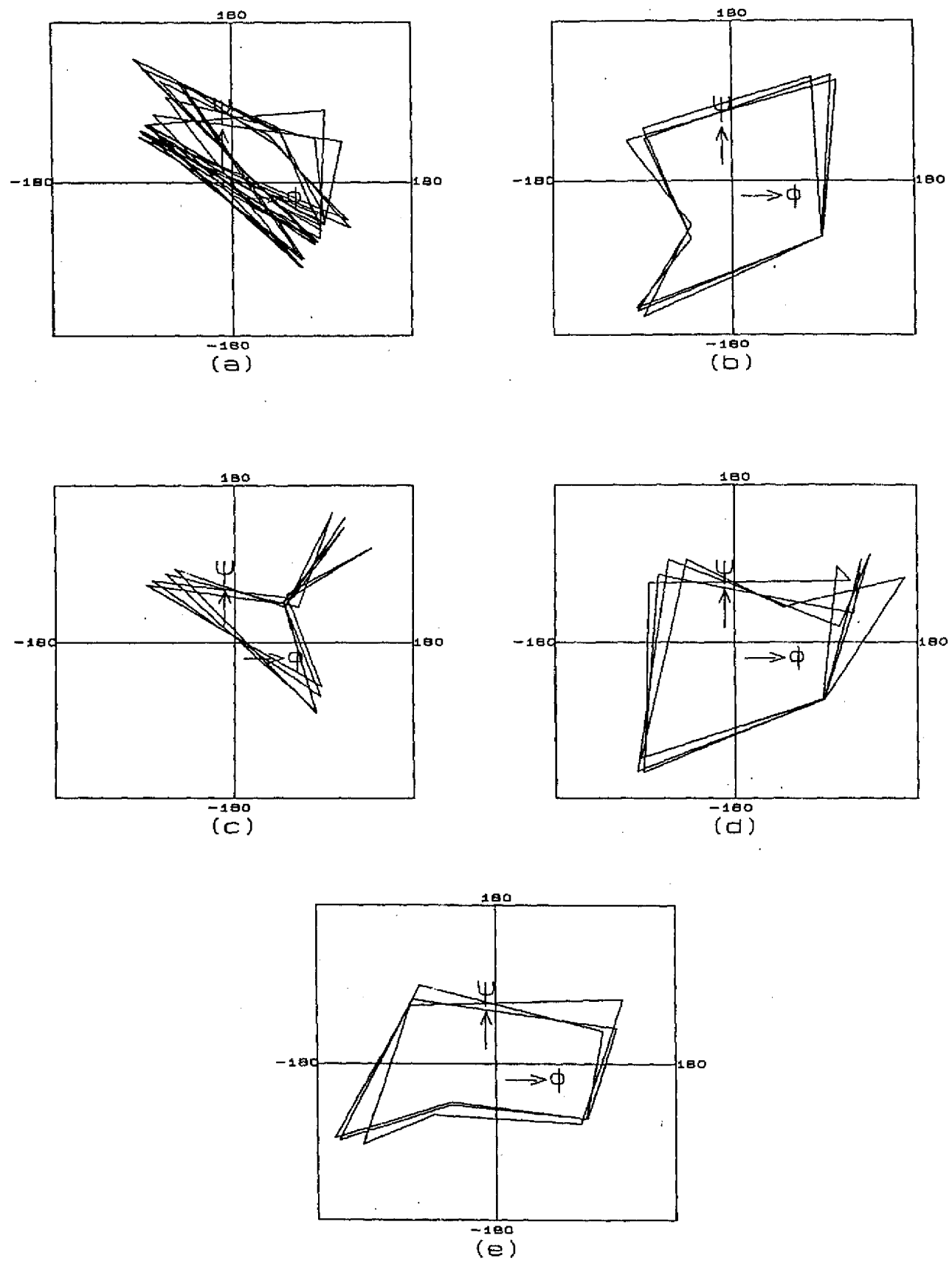

Figure 6. Superposition of the line diagrams of the MECs in the five Conformational families viz; , A, B, C, D and E. 
different turn structures which can be formed in local region of the 10-dimensional $(\varphi, \psi)$ conformational space Furthermore, it is highly probable that for conversion of one MEC to another in the same family, it is only required to form or break certain hydrogen bonds other than the conserved $\gamma$-turn hydrogen bond.

\subsection{Preliminary comparison of the MECs with the solid state conformations of CPP}

The list of compounds studied by X-ray crystallography is given in table 4. The hydrogen bonded turn patterns as shown by their crystal structures are also given in column 2 of this table. From this table it can be seen that the backbone, in all these examples possesses either a $\beta$-turn hydrogen bond or a pair of a hydrogen bonds belonging to $\beta$-turn and $\gamma$-turns.

To make a comparison of the observed examples with the MECs it is desirable to find the MEC which is conformationally close to the observed one. The values of the 10-dimensional distances between the MECs and the observed examples have been computed using expression (2). The MEC that has the least value of of the distance is referred to as the "closest MEC". The list of such MECs is given in column 3 of table 4 and the hydrogen bonding pattern found in these MECs is given in column 4 .

By comparing the hydrogen bonding pattern of the observed examples (given in column 2) and that of the closest MECs (given in column 4) it can be seen that $\beta$-turn type occurring in the tripeptide fragment of the observed examples (which is either type II $\beta$-turn or its inverse) is the same as that found in the corresponding closest MECs. However, there are some differences in the hydrogen bonding pattern involving other hydrogen bonds. These are detailed below.

(i) For the first two examples [c(Gly-Pro-Gly-D-Ala-Pro) and c(D-Phe-Pro-Gly-D la-Pro)] the closest MEC has two additional hydrogen bonds (type I' $\beta$ turn across the linking site and $\gamma^{\prime}$ hydrogen bonding across of these two hydrogen bonds $\gamma^{\prime}$-turn hydrogen bond is present in both the observed examples. However, type I' $\beta$-turn is ruled out in them because of the presence of Pro at $\mathrm{C}_{5}^{\alpha}$.

(ii) For the third and fourth examples [c(Pro-Gly-Pro-Ser-D-Ala) and c(Pro-Sar-MeAla-Thr-D-Val)] the closest MEC has two additional hydrogen bonds (type I $\beta$-turn across the linking site and $\gamma$ hydrogen bonding across of these two hydrogen bonds $\gamma$-turn is ruled out in them because of the presence of Pro at In addition to this the type I $\beta$-turn is also absent in both the examples, (iii) For the fifth example MEC11 which is the closest MEC has a $\gamma$-turn around $\mathrm{C}_{1}^{\alpha}$ which is ruled out in the observed case c(Gly-Pro-D-Phe-Gly-Ala) due to the presence of Pro at $\mathrm{C}_{2}^{\alpha}$

(iv) For the last example there is an extra hydrogen bond of the type $\gamma$ around the linking site $\mathrm{C}_{4}^{\alpha}$ in MEC9' which is absent in the observed example.

From table 4 it is clear that the observed examples form a sub set of the 23 MECs obtained in this study. In all the observed examples the backbone has adopted either type II $\beta$-turn or its inverse type. This peculiar bias of the backbone to adopt a $\beta$-turn of only one type (or its inverse) is rather surprising since the results of this study show that the backbone can adopt type I $\beta$-turn (or its inverse) with equal ease- In fact a few CPPs in solution have been shown to have conformations with type I $\beta$-turn (Kessler and Evermann 1982; Kessler and Kogler 1983; Stradley et al 1990; Rizo et al 1991). 


\section{Conclusions}

The information presented in table 4 is only a preliminary comparison of the MECs and the solid state conformations. The fact that the observed conformations are closer to one of the MECs shows that the MECs can usefully serve the purpose of a database of prototype conformations of the CPP backbone- Secondly, the fact that some of the hydrogen bonds present in the MEC are absent in the observed conformation shows that some hydrogen bonds can be sacrificed to relieve short contacts due to non-glycyl residues at the $\alpha$-carbon atoms as well as to bring about better close packing in the solid state. When one introduces the residues of the observed sequence in the MEC and carries out further energy minimization it is possible to obtain MECs which resemble the observed conformations more closely. The details of such a study will be presented elsewhere.

\section{References}

Balsubramanian R, Chidambaram K and Ramachandran G N 1970 Potential functions for hydrogen bond interactions. II- Formulation of an empirical potential function; Biochim. Biophys. Acta 221 196-206

Bara Y A, Friedrick A, Kessler H and Motler H 1978 1H and 13C-NMR Untersuchungen zur konformation von cyclo ((Phe)3-(G!y)2); Chem Ber 111 1045-1057.

Davidon W C 1959 Variable metric method for minimization; in $A \cdot E \cdot C \cdot$ Research and Development Report ANL-5990 (Rev·)

Demel D and Kessler H 1976 Conformation of cyclo(Pro-Phe-Gly-Phe-Gly); Tetrahedron Lett. 2801-2804.

Fletcher R and Powell M J D 1963 A rapidly convergent descent method for minimization; Comput J.

6 163-168

Gonnella N C, Zhang X· Jin Y, Om Prakash, Paris C G, Kolossvasy I, Gvida W C, Bohaceek R S, Vlattas I and Sytwa T 1994 Solvent effects on the conformation of cyclo(-D-Trp-D-Asp-Pro- D-Val-Lcu-). An NMR spectroscopy and molecular modeling study; Int J. Jeptide Protein Res. 43 454-462

Karle I L 1978 Crystal structure and conformation of cyclo(glycylprolylglycyl-D-alanylprolyl) containing $4 \rightarrow 1$ and $3 \rightarrow 1$ intramolecular hydrogen bonds; $J \cdot A m \cdot$ Chem $\cdot$ Soc $\cdot 100$ 1286-1289

Karle I L 1979 Conformation of cyclic pentapeptide Gly-L-Pro-L-Ser-D-Ala-L-Pro in the crystalline state and an example of rotational isomerism between analogues; J. Am Chem $\cdot$ Soc 101 181-184

Karle I L 1981 Conformation of cylic pentapeptides in the crystalline state: Cyclic(D-Phe-L-Pro-GlyD-Ala-L-Pro) $3 \rightarrow 1$ and $4 \rightarrow 1$ intramolecular hydrogen bonds; in Perspectives in peptide chemistry (eds) T Wieland, R Geiger and A Eberle (Basel: Karger) pp 261-271

Karle I L 1986 Variability in the backbone conformation of cyclic pentapeptide; Int J. Peptide Protein Res. 28 420-427

Kessler H 1982 Conformation and biological activity of cyclic peptides; Angew. Chem. Int Ed. Engl. 21 512-523

Kessler H and Evermann V 1982 Peptide conformations 24. Homo and heteronuclear 2D NMR spectro scopy of cyclic pentapeplides containing (he active sequence of somatostatin; Tetrahedron Lett. 23 4689-4692

Kessler H, Haupt A and Will M 1989 Design of conformationally restricted cyclopeptides for the inhibition of cholate uptake of hepatocytes; in Computer-aided drug design-methods and applications (eds) T J Perum and C-L-Propst (New York: Marcel-Dekker) pp 461-480

Kessler H and Holzemann G 1981 Cycloenkephaline-Synthese und konformations Studien; Liebigs Ann. Chem $\cdot 2028-2044$

Kessler $\mathrm{H}$ and Koggler $\mathrm{H} 1983$ Peptid konformation 26. Ein und zweidimensionale 1H-NMR unersuchungen zur konformation der beiden cycli sehen pentapeptide cyclo(Pro-Phe-Gly-Ser-Gly) und cyclo(Pro-Ser-Gly-Phe-Gly); Liebigs Ann· Chem· 316-329

Kessler H and Kondor P 1979 Peptide Konformation VI- Festlegung von intramolekularen Wasserstoff bsucken in cyclo(Phe-Gly-Xxx-Val-Ala) durch $1 \mathrm{H}$ NMR specktroskopic; Chem $\cdot$ Ber 112 3538-3551

Kessler H and Kutscher B 1985 Synthesis and NMR investigation of cyclic pentapeptide analogues of thymopoietins; Tetrahedron Lett. 26 177-180 
Lautz J, Kessler H, Boelens R, Kaptein R and van Gunsteren W F 1987 Conformational analysis of a cyclic thymopoietin-analogue by 1 H NMR spectroscopy and restrained molecular dynamics simulations; Int $J \cdot$ Peptide Protein Res. 30 404-414

Liu Z P and Gierasch L M 1992 Combined use of molecular dynamics simulations and NMR to explore peptide bond isomerisation and multiple intramolecular hydrogen bonding possibilities in cyclic pentapeptide, cyclo(Gly- Pro- D- Phe- Gly-Val); Biopolymers 32 1727-1739

Manjula G 1976 Theoretical studies on the conformations of cyclic peptides, Ph.D. Thesis- Indian Institute of Science, Bangalore

Manjula G and Ramakrishnan C 1979 Stereochemical studies on cyclic peptides: Part X- Conformational analysis of hydrogen bonded cyclic pentapeptides; Int $\cdot J \cdot$ Peptide Protein Res 13 353-362

Mauger A B, Staurt O A, Highet R J and Silverton J V 1982 Synthesis of an actinomycin-related peptide, CycIo(Thr-D-Val- Pro- Sar-MeAla), and conformational studies by nuclear magnetic resonance and X-ray crystallography; $\mathrm{J}$. Am $\cdot$ Chem $\cdot$ Soc $\cdot 104$ 174-180

Nagarajaram H A and Ramakrishnan C 1991 Energy minimization studies on cyclic pentapeptide; Indian $J \cdot$ Phys. B6S 449-454

Nagarajaram H A, Paul P K C, Ramanarayan K, Soman K V and Ramakrishnan C 1992 Conformational studies on $\beta$-bend containing a cis peptide unit; Int J. Peptide Protein Res. 40 383-394

Ovchinnikov $\mathrm{Yu} \cdot \mathrm{A} \cdot$ and Ivanov V T 1982 The cyclic peptides: structure, conformation and function; Peptides 5 307-642

Paul P K C and Ramakrishnan C 1985 Studies on hydrogen bonds. Part V-Hydrogen bonding in energy minimization studies of peptides; J. Biomol $\cdot$ Struct $\cdot$ Dyn $\cdot 2$ 879-898

Paul P K C and Ramakrishnan C 1987 Stereochemical studies on cyclic peptides: Part XIII- Energy minimization studies on cyclic hexapeptides having hydrogen bonds; Int J. Peptide Protein Res. 29 433-454

Ramakrishnan C, Ramanarayan K and Manjula G 1987 Stereochemical studies on cyclic peptides: Part XIV Conformational analysis of cyclic tripeptides Int $\cdot J \cdot$ Peptide Protein Res 29 657-671

Ramakrishnan C and Rao B N N 1980 Stereochemical studies on cyclic peptides: Part XI- Conformation of cyclic pentapeptides having intramolecular $3 \rightarrow 1$ hydrogen bonds; Int $J$. Peptide Protein Res. 15 $81-95$

Ramakrishnan C and Sarathy K P 1969 Stereochemical studies on cyclic peptides. Part IV. Conformational analysis of cyclopenlapeptides; Int J. Peptide Protein Res. 1 63-71

Ramachandran G N and Sasisekharan V 1968 Conformation of polypeptides and protein; Adv Protein Chem. 23 283-438

Rao B N N 1982 Conformational studies on cyclic pentapeptides, Ph. D. Thesis, Indian Institute of Science, Bangalore

Rizo J, Dhingra M M and Gicrasch L M 1991 Peptide models for reverse turns. The role of Asn in the $\mathrm{i}$ position of a $\beta$-turn; in Molecular conformation and biological interactions; $G$. $N$. Ramachandran Festschrift (eds) P Balaram and S Ramaseshan (Bangalore: Indian Academy of Sciences) pp 469-496

Rose G D, Gierasch L M and Smith J A 1985 Turns in peptide and proteins; Adv Protein Chem 37 1-109

Stradley S J, Rizo J, Brusch M D, Stroup A N and Gicrasch L M 1990 Cyclic peptides as models for reverse turns: Determination of the equilibrium distribution between type I and type II conformations of Pro-Asn and Pro-Ala $\beta$-turns; Biopolymers 29 263-287

Stroup A N, Rockwell A L and Gierasch L M 1992 Solution conformation of two cyclic pentapeptides: Cyclo(Gly- Pro- D- Phe- Gly-Ala) and Cyclo(Gly- Pro- D- Phe- Gly-Val); Biopolymers 32 1718-1725

Stroup A N, Rheingold A L, Rockwell A L and Gierasch L M 1987 Crystal structure of cyclo(Gly-L-Pro-D-Phe-Gly-L-Val): An example of a new residue type of three-residue turn; $\mathrm{J}$. $\mathrm{Am}$. Chem Soc. 109 7146-7150 